\title{
Seeing What Lies in Front of Your Eyes: Understanding and Insight in Teaching and Research
}

\author{
Elena Popova, Valentin L. Popov, and Alexander E. Filippov
}

\begin{abstract}
In the present paper, we considered the phenomena of understanding and discoveries (as a sort of "social understanding") and found that the empirical properties of these phenomena (the critical character and emerging of a new property) have much in common with first-order phase transitions. From this point of view, we discuss both the process of understanding and discoveries and the reasons impeding "seeing what lies in front of our eyes". In our opinion, these ideas can be further studied on the same phenomenological basis, without detailed understanding of the underlying neuronal mechanisms.
\end{abstract}

Keywords Understanding $\cdot$ Insight $\cdot$ Discovery $\cdot$ Phase transitions $\cdot$ Order parameter $\cdot$ History of science $\cdot$ Friction $\cdot$ Contact $\cdot$ Adhesion

\section{Introduction}

This paper is devoted to a phenomenon of fundamental importance for many areas of human activity, including research and teaching - the phenomenon of understanding. It is clear that teachers must ensure that the material they mediate is understood and not only memorized or "only" practiced well. However, this central phenomenon of learning always remains a mystery. The act of understanding can be compared to that of "seeing": When understanding something, we suddenly see "the picture as a whole". However, this is not at all easy-as Goethe once said: "The hardest thing to see is what lies in front of your eyes".

\footnotetext{
E. Popova $(\varangle) \cdot$ V. L. Popov

Technische Universität Berlin, Berlin, Germany

e-mail: elena.popova@tu-berlin.de

V. L. Popov

e-mail: v.popov@tu-berlin.de
}

\author{
A. E. Filippov \\ Donetsk Physico-Technical Institute, Donetsk, Ukraine
}


Based on historical examples, we suggest considering the mechanism of "sudden understanding" as a first-order-phase transition in the cognitive space (individual understanding) or a combination of cognitive and real space (discoveries).

\section{Phenomenon of Understanding}

The paradoxical truth expressed by Goethe is valid both for the scientific perception of the world and for everyday life. Consider the painting "Gartenlokal an der HavelNikolskoe" (Fig. 1).

When people stand in front of this painting, showing a Cafe at one of the lakes in Berlin, they usually do not see anything strange in it-even if they study the painting attentively. There are just people sitting in a cafe and speaking to each other. What goes unnoticed is the fact that none of the chairs in this painting has legs: Everyone is sitting in the air! It is still a matter of debate whether Liebermann just did not have enough time and the painting is incomplete or this was his joke with the observer, but the fact is that the majority of spectators are not at all disturbed by the missing details. They just do not see that they are missing!

In this case, the details we do not see are not important for the correct perception of the painting. However, sometimes it is exactly the details we overlook that are important. In the same way, something that may be hard to understand initially, can

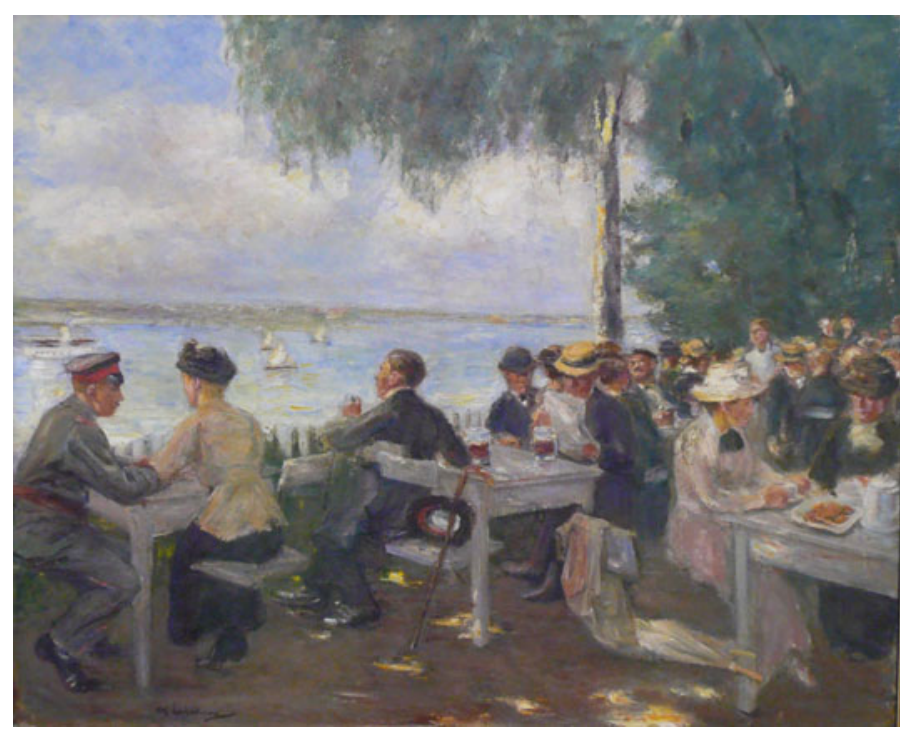

Fig. 1 Max Liebermann, Garden Restaurant on the Havel—Nikolskoe, 1916, Berlin, Nationalgalerie 
Prof. Popov | Colloquium Advanved Mechanics | 28.04.2017

Problem 1. Two tractors pull a box with ropes (see picture). The velocities of the tractors are directed along the ropes and are $\boldsymbol{v}_{1}$ and $\boldsymbol{v}_{2}$. The angle between the ropes is alpha. What is the velocity of the box and how is it directed?

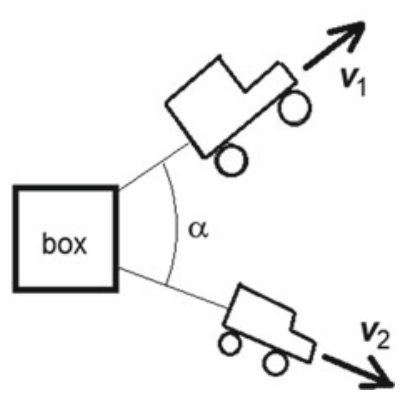

Fig. 2 A task from the "Colloquium Advanced Mechanics" at the TU Berlin

become trivial once seen from the right perspective: The solution lies in front of our eyes, but we do not see it.

One of the authors of this paper (V.P.) offers a course at the TU Berlin that is specifically designed to illustrate and to train the ability to understand: the "Colloquium for Advanced Mechanics". The typical feature of the tasks that are handled in the Colloquium is that they are "very difficult problems, that are very easy to solve". The statement is contradictory only at first sight. The tasks of the Colloquium often cannot be solved by students, but only because they do not approach them from the correct perspective.

Here is an example of a task from the Colloquium (Fig. 2). Two tractors pull a box with ropes. The velocities of the tractors are directed along the ropes and are $v_{1}$ and $v_{2}$. The angle between the ropes is $\alpha$. What is the velocity of the box and how is it directed? The task sounds simple. It is clear to everybody that it must have a solution: If you pull a box, it will have to move somewhere. And yet it is a task that even scientific collaborators at the University cannot solve immediately. The first idea that comes to mind is to sum up the velocities of the tractors. However, at least after considering the special case of parallel ropes and equal velocities, one can see that this idea is unfortunately completely wrong.

Experience shows that only exceptional personalities can solve this problem quickly and correctly-in spite of the fact that the task is solvable in two lines! There is just nothing to "calculate" in this problem! The only thing one needs is to look at the problem from the correct point of view. Once one understands the underlying principle, one can only say: "Ah!" And that $i$ s the solution.

The colloquium essentially consists of tasks of this kind, in which one finds the solution not by "working long and hard", but by real understanding. As Goethe has said, the hardest thing to see is what lies in front of your eyes! 


\section{Discoveries in the History of Science as "Seeing the Obvious"}

A similar phenomenon of understanding is known in the history of science. History is full of examples where great discoveries were nothing more than seeing what lies in front of your eyes [1].

Let us take fracture theory as an example. In 1921, Alan Griffith put forward an idea why the strength of materials is much lower than the theoretical one and why it depends on the size of engineering parts [2]. His paper was the beginning of the theory of fracture. But what did Griffith really do? He only said that the crack tip is in equilibrium if the change of energy due to a small displacement is zero. This energy change consists of the relaxation of elastic energy and the work of separation of surfaces. One of them is positive and the other negative. If their absolute values are equal then the crack is in equilibrium. This is of course nothing else than the principle of virtual work for mechanical systems. This principle is by no means an invention of Griffith, it was already known to d'Alembert and Leonard Euler. It is a standard, well-known equilibrium condition, which can be found in any introductory textbook on mechanics. New was only that Griffith applied this old principle to the crack. Interestingly, he did not even have to carry out any complicated analytical calculations, because the elastic energy, which is released due to a small movement of a crack tip, was already known. The corresponding problem was solved in 1911 by Inglis [3], and the expression for the work of adhesion is trivial: it is the product of the specific work of adhesion and the new surface area produced by the crack opening. Thus, Griffith equated two contributions, which were both known at that time and thus produced the famous, classical results of fracture theory!

50 years later, Johnson, Kendall and Roberts (JKR) published their famous paper on adhesion, one of the most cited papers in the field of contact mechanics [4]. In their paper, JKR note that their approach is equivalent to that of Griffith. They write: "...the approach followed in this analysis, is similar to that used by Griffith in his criterion for the propagation of a brittle crack." As a matter of fact, JKR realized that the adhesive contact is the inverted Griffith crack (in the crack the discontinuity is mostly inside and in the "adhesive contact" outside). After that, JKR applied the principle of energy balance exactly in the way Griffith did. The only difference is in the expressions for the elastic energy. Griffith used the energy of an "internal crack" provided by Inglis and JKR used the expressions provided by Hertz [5] and Boussinesq [6]. The realization of the equivalence of the problem of adhesive contact to the problem of the Griffith crack was the main contribution of JKR, as a matter of fact, their only contribution. Already Griffith had all the necessary ingredients for the solution of the adhesive contact problem, he merely had no need to solve it. If he had, we probably would have had the "JKR"-solution already in 1921. It is surprising that 50 years were needed just to recognize the equivalence of these two problems!

But the history of adhesion does not end here. One of the most effective numerical simulation methods in contact mechanics is at the present the Boundary Element Method (BEM), which allows simulation of arbitrary contact configurations [7]. 


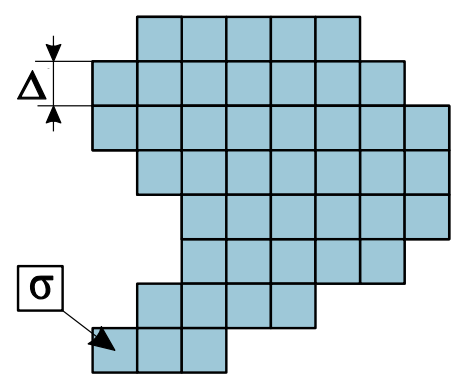

a

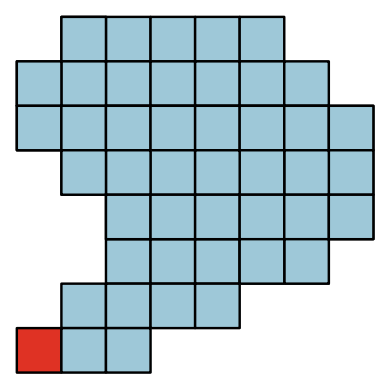

b

Fig. 3 In each calculation step, stress in each particular discretization element is determined. If the stress $\sigma$ in a given element at the boundary of the contact area exceeds the critical value (3), it is "detached" and the stress in this element is set zero

However, only in 2015 an idea was put forward on how to simulate adhesive contacts in the framework of BEM: The use of a simple, unmodified Griffith criterion for the BEM detachment condition [8]. Let us explain this in more detail.

In each iteration of the BEM simulation, the stress in each discretized cell is determined and then it is decided whether this cell is still in contact or not. For non-adhesive systems, the answer is very simple: The pressure must be positive. But if you have an adhesive contact then the pressure can become negative and we have to find a criterion for when a particular element will lose the connection to the counter-body. In 2015, two scientists suggested applying the Griffith criterion: Let us assume that the contact of a cell is lost, then the stress in this cell vanishes (Fig. 3). It is easy to calculate analytically what amount of elastic energy is relaxed due to the stress vanishing:

$$
\Delta U_{e l}(\tau)=\kappa \frac{\sigma^{2}}{E^{*}} \Delta^{3}
$$

with

$$
\kappa=\frac{2}{3 \pi}\left(1-\sqrt{2}+\frac{3}{2} \log \left(\frac{\sqrt{2}+1}{\sqrt{2}-1}\right)\right) \approx 0.473201 .
$$

The element is in the state of indifferent equilibrium if the change of elastic energy is equal to the work of adhesion needed for creating the free surface with the area $\Delta^{2}, \Delta U_{a d h}=\gamma_{12} \Delta^{2}$, or with (1), $\kappa \sigma^{2} \Delta^{3} / E^{*}=\gamma_{12} \Delta^{2}$, where $\gamma_{12}$ is the work of adhesion per unit area. For the critical detachment stress they obtained

$$
\sigma_{c}=\sqrt{\frac{E^{*} \gamma_{12}}{0.473201 \cdot \Delta}}
$$


It is now assumed that if this elastic energy is equal to the work of separation then the element will detach. In other words, as soon as the elastic energy is enough for creating new surfaces, they will be created. This solution is so simple and straightforward that one can only ask why it took another 44 years to apply the Griffith criterion in numerical simulation!

The present paper is not devoted to any detailed substantiation of ideas, let us just mention that the simple criterion presented above reproduces all known analytical solutions exactly [9].

\section{Understanding as Changing the Point of View}

The above examples illustrate very clearly the idea that the real mechanism behind new theories and paradigms is often a transfer of knowledge from one subject area to another [1]. A similar process in individual perception is changing the point of view. But what exactly does that mean? This mechanism reflects fundamental philosophical roots of the term "meaning" that were elaborated in detail in the second half of the twentieth century, owing to the development of system analysis and increased interest in the nature of complicated systems. According to system analysis, properties of a system cannot be understood by looking on it from inside the system [10]. Recall the definition of a system given by R. Ackoff: "A system is a whole which is determined by its function in the system, a part of which it is" [11]. Therefore, understanding of the meaning, purpose, and objectives of any system can be achieved only at the level of the containing system (supersystem). This means that true understanding implies the placing of a given object into a corresponding supersystem where it plays a specific role. Depending on the supersystem, the same object can have different meanings.

The above historical examples have one thing in common: The "seeing" occurs by exchanging the supersystems. Very often, one hears that scientists and engineers have to "think outside the box". From the point of view of system analysis, thinking outside the box becomes a generic, compulsory prerequisite to any true understanding.

Of interest is R. Ackoff's comment on the relationship between the analysis of a part of a system and the supersystem: "Note that an analysis starts with division of a subject into parts and gives knowledge. Synthesis starts with combination of things and gives understanding. Analysis is the way the scientists investigate. Synthetic thinking is manifested in designing... There is one more extremely important aspect of synthetic thinking. The systems we deal with are becoming increasingly sophisticated. Scientists seek the effective ways of treating them. Unfortunately, most of them approach the problem analytically. As a result, they introduce so many variables and relations between them that we cannot cope with them. However, if complexity is dealt with in a synthetic way, by designing, like designing a high-rise building or a city, there is no level of complexity that we cannot effectively cope with" $[10,11]$.

If we accept the viewpoint of Ackoff, then we have to accept that understanding is the final result of a synthetic activity aimed at a broadening the context in which the 
given object is considered or at trying a large variety of "contexts" until a "correct" one is found.

There is another potentially important point of this process, which is best illustrated not on examples of personal perception but on the history of discoveries: It is not always clear that the correct supersystem has already been found, so that the final solution may require many attempts. In the history of science, this is seen by the very widespread phenomenon of multiple discoveries.

\section{Multiple Discoveries}

Very often scientific results are obtained multiple times- "re-discovered" without knowing the predecessors. Take as an example the history of the so-called Method of Dimensionality Reduction [12]. This is a method of representing contact mechanics in a simple way that can be taught even to undergraduate students and can be used by any practical engineer. It was developed in the decade from 2005 to 2015 . However, it was invented already much earlier and reinvented many times in the course of history. And each time, people just did not see what was lying in front of their eyes. It was first formulated in a paper by Schubert in 1942 in Germany [13]. The same solution was found by Galin in 1946 in Russia [14] and later by Green and Zerna in 1954 [15]. Sneddon translated the book of Galin from Russian into English in 1950th (see a later publication [16]) and also published 1965 a very influential paper which contains exactly the same solution, since then mostly known as the Sneddon solution [17]. In 1998 Jäger suggested an alternative physical interpretation of the same equations [18]. Finally, they were reformulated as a simple mnemonic rule and generalized to a large variety of contact problems in 2005 to 2015, thus creating MDR $[10,12]$. It is striking that it took about 70 years to realize that the solution lies in front of our eyes!

\section{Understanding as a Phase Transition}

The sudden character of understanding shows that this is a critical phenomenon. A prerequisite for it is a synthetic (design) work, but the final act is often perceived as "instantaneous". Understanding is bringing order in a large number of elements. It can thus be interpreted as a "phase transition" establishing a "long range order" in the corresponding "cognitive space". This transition can be easily followed phenomenologically by considering an impressionist painting from various distances. When considered in the vicinity, the painting looks like a chaotic set of color spots (Fig. 4). Going further from the picture, one suddenly recognizes the picture as a whole. Interestingly, this process is almost reversible so that one can repeat this "act of understanding" or "act of recognition" many times. At this point, we cannot discuss the question of the exact neuronal mechanism of this phase transition. We just use 


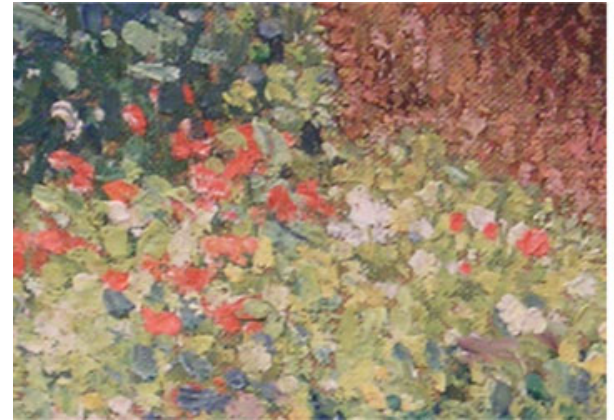

a

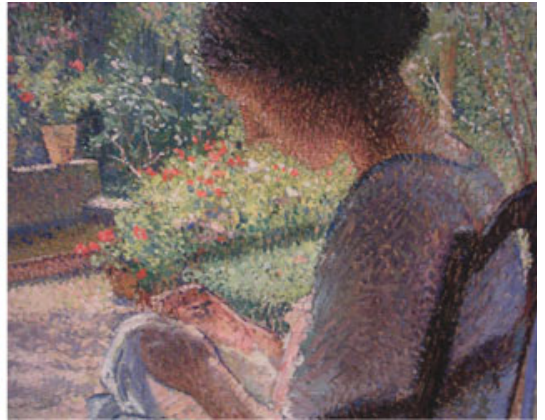

b

Fig. 4 Painting "Lisette Sewing in front of the Entrance Door of Marquerol" by Henri Martine: a view from vicinity, $\mathbf{b}$ view of the whole picture. Tel Aviv museum of Art

the finding of the theory of phase transitions, which says that local interactions in a distributed system can lead to a "sudden" establishment of a long-range order in the system when interactions achieve a critical value [19]. This change can lead to the appearance of qualitatively new properties, such as superconductivity. In the human perception we see empirically similar sudden state changes with the emerging of a new property - that of "understanding". It surely would be an intriguing and fruitful task to try to find out what exactly the neuronal mechanisms of this transition are, which could greatly facilitate the development of didactics.

The analogy of understanding and seeing brings another important aspect into play.

\title{
7 Interrelation of Personal Understanding and Discoveries
}

\author{
The fastest runner doesn't always win the race, \\ and the strongest warrior doesn't always win the battle. \\ The wise sometimes go hungry, \\ and the skillful are not necessarily wealthy. \\ And those who are educated don't always lead successful lives. \\ It is all decided by chance, \\ by being in the right place at the right time. \\ Ecclesiastes 9:11
}

While the acts of personal understanding and scientific discovery have much in common, one has to also see the essential difference between both. The above example of the history of the MDR shows that "understanding" in a historical context does not merely mean that something is seen by an individual scientist. It is important 
that the act of seeing a scientific result coincides with the act of public recognition of its importance: Understanding "not at the right time" "does not count". Only "collective seeing" is a true discovery. We often do not really know how many times the same scientific truth was re-discovered and intensive historical research is needed to answer the question: "who was the first"? Maybe one can speak of the act of "public understanding", which means understanding reaching a large (relevant) community. However, it is very difficult to know in advance whether a particular result will be broadly accepted by scientific community. That is why it is so difficult to make "discoveries on demand"- - in reality, discoveries are in most cases understood as such a posteriori-in rare lucky cases of (later) public recognition.

The analogy with phase transitions leads to the conclusion that "authorship" of particular discoveries is relative. If the medium is far from the critical state, no fluctuations will create the long order parameters, and vice versa, if it is in the critical or overcritical state, then any nucleus will lead to the phase transition. Can we blame those researchers who by chance did not work in some field which the future would show to be very important and, on the contrary, praise those who for some reason were "centuries ahead of their time" (in most cases without knowing this)?

For success of a personal scientific career, it is very important to work on problems which may have a public resonance (at least in the relevant scientific community). The highest scientific qualification lies exactly in the general orientation of what problems are "of interest".

However, for the progress of science as a whole, this personal success is of no relevance. The true precondition of phase transitions is the local interaction. Preparation of this local interaction is as important for science as being an "ingenious inventor" (the latter often meaning to be a random "fluctuation" at the right time and at the right place.)

\section{What Prevents Us Seeing What Lies in Front of Our Eyes?}

The analogy of the phenomenon of understanding with phase transitions suggests a solution to the question of what prevents us from seeing what lies in front of our eyes. The fact that the "act of understanding" often occurs suddenly, implies that the underlying phase transition is a first-order phase transition. This means that the transition always occurs through formation of initially small nuclei, which later expand to the whole phase space. This propagation is connected with some "friction" in the boundary line. A very similar phenomenon takes place in social processes. A society of sufficiently large volume (including the scientific society as well) causes a practically infinite barrier for the immediate acceptance of new knowledge, even if it was already achieved by an individual researcher. Thus, the factor impeding the 
understanding is a resistance to the change of state. This resistance is a generic property of any first-order-phase transformation. What is sometimes called the "inertia of thinking" is in reality a "friction of thinking". The essential question is of course what factors determine this "friction". Just as for example ferromagnetic materials can have a large or small internal friction for the motion of phase boundaries (corresponding to hard and soft magnetic materials), the resistance to understanding may be higher or lower depending on factors which are not yet understood. Their determination could greatly facilitate didactics.

Similarly, discoveries can be considered as acts of "social understanding". They also occur "suddenly" (on the time scale of historical processes) and thus have the features of first-order transitions. The phase space now contains both "cognitive dimensions" and the real space dimension (in form of a geographic distribution of researchers). Any nucleus of new understanding will have to overcome the frictional force for boundary propagation. In everyday language, one can say that anybody offering a radically new idea will stumble upon a consolidated resistance by the whole system of knowledge accumulated and lovingly ordered on the shelves.

In the theory of first-order transitions it is known that a high boundary friction leads to the necessity of "overheating" the system to initiate the transition. In our analogy with understanding, this means that one has to do much more work and to accumulate more information related to the particular topic in the space of knowledge than it is necessary to perform "act of understanding" itself.

Another property of the first-order transition is the existence of the threshold of "absolute instability". In our analogy, this means that at some point it becomes impossible to ignore new knowledge and the transition becomes unavoidable.

The nuclei of a new phase can also dissolve again if the systems moves away from the critical point. The history of civilization provides many examples of the reverse process when already achieved knowledge "suddenly disappears", if for some reason it looses its social importance.

\section{Conclusion}

In the present paper, we considered the phenomena of understanding and discoveries (as a sort of "social understanding") and found that the empirical properties of these phenomena (the critical character and emerging of a new property) have much in common with first-order phase transitions. In our opinion, these ideas can be further studied on the same phenomenological basis, without detailed understanding of the underlying neuronal mechanisms - similarly to the famous treatment of the theory of superconductivity in the phenomenological theory of phase transformations [20].

However, just as a statistical physics give a deeper insight into the physical understanding of phase transitions, understanding the neuro-physiological mechanisms of perception and handling of "newness" by our brain would strongly facilitate also the phenomenological view on understanding. Some important findings in this relation can be found in [10]. 
In philosophy, this picture has already been developed over centuries under the notion of "measure" as a unity of quantity and quality (Hegel) [21]. However, we find that the picture provided by the theory of phase transitions provides more details and more understanding of the underlying processes.

The true understanding is the only quality, which enables a person or a community to make real progress in any branch of science or engineering. The analogy of understanding to a long range order provides an (we hope useful) illustration of what sometimes is called "complete knowledge" [10], meaning that understanding implies seeing the "picture as a whole". The long range order is exactly what provides a picture the "wholeness".

Acknowledgements The authors are grateful to Prof. Leonid Evgenyevich Popov and Emanuel Willert for inspiring and useful discussions and Nikita Popov for proof reading the Chapter.

\section{References}

1. Popova E, Popov VL (2018) Note on the history of contact mechanics and friction: Interplay of electrostatics, theory of gravitation and elasticity from Coulomb to Johnson-Kendall-Roberts theory of adhesion. Phys Mesomech 21(1):1-5

2. Griffith AA (1921) The phenomena of rupture and flow in solids. Philos Trans R Soc A: Math Phys Eng Sci 221:582-593

3. Inglis CE (1913) Stresses in a plate due to the presence of cracks and sharp corners. Trans Inst Naval Arch 55:219-241

4. Johnson KL, Kendall K, Roberts AD (1971) Surface energy and the contact of elastic solids. Proc R Soc Lond A 324(1558):301-313

5. Hertz H (1881) Über die Berührung fester elastischer Körper. Journal Für Die Reine Und Angewandte Mathematik 92:156-171

6. Boussinesq J (1885) Applications des Potentiels a l'Etude de l’Equilibre et du Mouvement des Solides Elastiques. Gauthiers-Villars, Paris

7. Pohrt R, Li Q (2014) Complete boundary element formulation for normal and tangential contact problems. Phys Mesomech 17(4):334-340

8. Pohrt R, Popov VL (2015) Adhesive contact simulation of elastic solids using local meshdependent detachment criterion in Boundary Elements Method. Facta Univ Ser: Mech Eng 13(1):3-10

9. Popov VL, Pohrt R, Li Q (2017) Strength of adhesive contacts: influence of contact geometry and material gradients. Friction 5(3):308-325

10. Popov LE, Postnikov SN, Kolupaeva SN, Slobodskoy MI (2015) Natural resources and technologies in educational activities: education in times of accelerated technological development. Cambridge International Science Publishing, Cambridge, p 133

11. Ackoff R, Greenberg D (2008) Turning learning right side up: putting education back on track. Wharton School Publishing, New Jersey, p 224

12. Popov VL, Heß M (2015) Method of dimensionality reduction of contact mechanics and friction. Springer, Berlin

13. Schubert G (1942) Zur Frage der Druckverteilung unter elastisch gelagerten Tragwerken. Ingenieur-Archiv 13(3):132-147

14. Galin LA (1946) Three-dimensional contact problems of the theory of elasticity for punches with circular planform. Prikladnaya Matematika I Mekhanika 10:425-448 ((in Russian))

15. Green AE, Zerna W (1954) Theoretical elasticity. Clarendon Press, Oxford 
16. Galin LA (1961) Contact problems in the theory of elasticity. North Carolina (USA): Department of Mathematics, School of Physical Sciences and Applied Mathematics, North Carolina State College

17. Sneddon IN (1965) The relation between load and penetration in the axisymmetric Boussinesq problem for a punch of arbitrary profile. Int J Eng Sci 3(1):47-57

18. Jaeger J (1995) Axi-symmetric bodies of equal material in contact under torsion or shift. Arch Appl Mech 65:478-487

19. Landau LD, Lifshitz EM (1980) Statistical physics, 3rd edn. Part 1, vol 5. ButterworthHeinemann, Oxford. ISBN 978-0-7506-3372-7

20. Ginzburg VL, Landau LD (1950) To the theory of superconductivity. In: Pis'ma v ZhTF, 20, 1064 (in Russian)

21. Hegel GWF (2010) Encyclopedia of the philosophical sciences in basic outline, part 1, science of logic. Cambridge University Press, Cambridge (Cambridge Hegel Translations)

Open Access This chapter is licensed under the terms of the Creative Commons Attribution 4.0 International License (http://creativecommons.org/licenses/by/4.0/), which permits use, sharing, adaptation, distribution and reproduction in any medium or format, as long as you give appropriate credit to the original author(s) and the source, provide a link to the Creative Commons license and indicate if changes were made.

The images or other third party material in this chapter are included in the chapter's Creative Commons license, unless indicated otherwise in a credit line to the material. If material is not included in the chapter's Creative Commons license and your intended use is not permitted by statutory regulation or exceeds the permitted use, you will need to obtain permission directly from the copyright holder. 